\title{
Hierarchical Decoupling Principle of a MIMO-CDMA Channel in Asymptotic Limits
}

\author{
Keigo Takeuchi \\ Graduate School of Informatics \\ Kyoto University \\ Kyoto, Japan \\ takeuchi@sys.i.kyoto-u.ac.jp
}

\author{
Toshiyuki Tanaka \\ Graduate School of Informatics \\ Kyoto University \\ Kyoto, Japan \\ tt@i.kyoto-u.ac.jp
}

\begin{abstract}
We analyze an uplink of a fast flat fading MIMOCDMA channel in the case where the data symbol vector for each user follows an arbitrary distribution. The spectral efficiency of the channel with CSI at the receiver is evaluated analytically with the replica method. The main result is that the hierarchical decoupling principle holds in the MIMO-CDMA channel, i.e., the MIMO-CDMA channel is decoupled into a bank of singleuser MIMO channels in the many-user limit, and each singleuser MIMO channel is further decoupled into a bank of scalar Gaussian channels in the many-antenna limit for a fading model with a limited number of scatterers.
\end{abstract}

\section{INTRODUCTION}

Direct-sequence spread-spectrum code-division multiple access (CDMA) has been utilized as a multiple access scheme in wireless communication. As a method of overcoming the capacity bottleneck in future wireless communication, multipleinput multiple-output (MIMO) systems have attracted so much attention since the latter half of the 1990s [1]. Recently, MIMO systems with CDMA technology (MIMO-CDMA) have been studied [2], [3].

Mantravadi et al. [2] evaluated the asymptotic spectral efficiency of a MIMO-CDMA channel with Gaussian modulation using the random matrix theory. However, other data modulation schemes such as quadrature phase shift keying (QPSK) modulation, or more generally, $M$-quadrature amplitude modulation ( $M$-QAM) are commonly employed in practice. Thus, it is important to analyze the MIMO-CDMA channel with a modulation of the kind. The purpose of our study is to evaluate the spectral efficiency of the MIMOCDMA channel in the case that the data symbol vector for each user follows an arbitrary distribution.

It was reported [4] that the spectral efficiency of a CDMA channel with binary phase shift keying modulation can be evaluated with the so-called replica method. Then, the method was applied to the performance evaluation of MIMO systems [5], [6], [7]. Guo et al. [8] analyzed a CDMA channel in the case that the data symbol for each user follows an arbitrary distribution and claimed that the decoupling principle, the equivalent result to which has already been proved in the case of Gaussian modulation [9], [10], holds in an asymptotic limit, i.e., the CDMA channel is decoupled into a bank of scalar Gaussian channels. This principle makes possible the analytical evaluation of the spectral efficiency since the degree of freedom drastically decreases. However, it is not still clear except special cases [2] whether or not the decoupling principle holds in the MIMO-CDMA channel.

In this paper, we claim that the decoupling principle holds in the MIMO-CDMA channel and evaluate the spectral efficiency of the channel using the replica method.

\section{MODEL}

We consider the uplink of a synchronous $K$-user MIMOCDMA flat fading channel [2]

$$
\boldsymbol{y}_{l}=\sum_{k=1}^{K} s_{l}^{k} \boldsymbol{H}^{k} \boldsymbol{x}^{k}+\boldsymbol{n}_{l},
$$

where the $k$ th user has $M_{k}$ transmit antennas and the receiver has $N$ receive antennas. $\boldsymbol{x}^{k}=\left(x_{1}^{k}, \ldots, x_{M_{k}}^{k}\right)^{T}$ is the data symbol vector for the $k$ th user and $\left\{s_{l}^{k} ; l=1, \ldots, L\right\}$ is the spreading sequence for the $k$ th user. We assume that $\left\{\boldsymbol{x}^{k} ; k=1, \ldots, K\right\}$ are mutually independent complex random variables and that the real part and the imaginary part of $\left\{s_{l}^{k}\right\}$ are independent and identically distributed (i.i.d.) zeromean random variables with the variance $1 / 2 L . \boldsymbol{H}^{k}$ represents the $N$-by- $M_{k}$ channel matrix for the $k$ th user, i.e., the ( $n$, $m_{k}$ )-element $h_{n m_{k}}^{k}$ of $\boldsymbol{H}^{k}$ is the channel gain from the $m_{k}$ th transmit antenna of the $k$ th user to the $n$th receive antenna. We assume that $\left\{\boldsymbol{H}^{k} ; k=1, \ldots, K\right\}$ are mutually independent. We consider the case that the noise is additive white Gaussian noise (AWGN), i.e., $\left\{\boldsymbol{n}_{l} ; l=1, \ldots, L\right\}$ are i.i.d. zero-mean circularly symmetric complex Gaussian random variables with the covariance matrix $\sigma^{2} \boldsymbol{I}_{N}$, which are denoted by $\mathcal{C N}\left(\mathbf{0}, \sigma^{2} \boldsymbol{I}_{N}\right) . \boldsymbol{y}_{l}$ represents the $N$-dimensional received signal vector.

We write the entire data symbol vector $\overrightarrow{\boldsymbol{x}}$ and the received signal vector $\overrightarrow{\boldsymbol{y}}$ in a symbol period as $\overrightarrow{\boldsymbol{x}}=\left(\boldsymbol{x}^{1^{T}}, \ldots, \boldsymbol{x}^{K^{T}}\right)^{T}$, and $\overrightarrow{\boldsymbol{y}}=\left(\boldsymbol{y}_{1}^{T}, \ldots, \boldsymbol{y}_{L}^{T}\right)^{T}$, respectively. The maximal sum rate in the fast fading channel with perfect channel side information at the receiver is given by the conditional mutual information (the base of logarithms is taken to 2 in this paper) between $\overrightarrow{\boldsymbol{x}}$ and $\overrightarrow{\boldsymbol{y}}$ conditioned on the spreading sequences and the channel matrices [1]

$$
I(\overrightarrow{\boldsymbol{x}} ; \overrightarrow{\boldsymbol{y}} \mid \mathcal{S}, \mathcal{H})=\mathrm{E}\left[\log \frac{p(\overrightarrow{\boldsymbol{y}} \mid \overrightarrow{\boldsymbol{x}}, \mathcal{S}, \mathcal{H})}{\mathrm{E}_{\overrightarrow{\boldsymbol{x}}}[p(\overrightarrow{\boldsymbol{y}} \mid \overrightarrow{\boldsymbol{x}}, \mathcal{S}, \mathcal{H})]}\right],
$$


where $\mathcal{S}=\left\{s_{l}^{k} ; l=1, \ldots, L, k=1, \ldots, K\right\}, \mathcal{H}=\left\{\boldsymbol{H}^{k} ; k=\right.$ $1, \ldots, K\}$, and where $p(\overrightarrow{\boldsymbol{y}} \mid \overrightarrow{\boldsymbol{x}}, \mathcal{S}, \mathcal{H})$ is given by

$$
p(\overrightarrow{\boldsymbol{y}} \mid \overrightarrow{\boldsymbol{x}}, \mathcal{S}, \mathcal{H})=\frac{1}{\left(\pi \sigma^{2}\right)^{L N}} \prod_{l=1}^{L} \mathrm{e}^{-\frac{1}{\sigma^{2}}\left\|\boldsymbol{y}_{l}-\sum_{k=1}^{K} s_{l}^{k} \boldsymbol{H}^{k} \boldsymbol{x}^{k}\right\|^{2}} .
$$

We define the spectral efficiency $\mathcal{C}_{\text {MIMO-CDMA }}$ as the maximal sum rate per chip and per transmit antenna. In the manyuser limit, where the number of users $K$ and the spreading factor $L$ tend to infinity with their ratio $\beta=K / L$ fixed, the spectral efficiency is given by

$$
\mathcal{C}_{\text {MIMO-CDMA }}=\frac{\beta}{\bar{M} \ln 2} \mathrm{E}_{\mathcal{H}}[\mathcal{F}]-\bar{\mu}^{-1} \log \left(\pi \sigma^{2} \mathrm{e}\right),
$$

where $\bar{M}=\lim _{K \rightarrow \infty} K^{-1} \sum_{k=1}^{K} M_{k}$ and $\bar{\mu}$ is the ratio of the average number of transmit antennas to the number of receive antennas, i.e., $\bar{\mu}=\bar{M} / N$. The free energy $\mathcal{F}$ is defined as

$$
\mathcal{F}=-\lim _{K, L \rightarrow \infty} \frac{1}{K} \mathrm{E}[\ln p(\overrightarrow{\boldsymbol{y}} \mid \mathcal{S}, \mathcal{H}) \mid \mathcal{H}] .
$$

\section{REPLICA ANALYSIS OF MIMO-CDMA}

We explain briefly the calculation procedure of the free energy (5) [4], [8]. Substituting the following identity:

$$
\lim _{u \rightarrow 0} \frac{\partial}{\partial u}[p(\overrightarrow{\boldsymbol{y}} \mid \mathcal{S}, \mathcal{H})]^{u}=\ln p(\overrightarrow{\boldsymbol{y}} \mid \mathcal{S}, \mathcal{H})
$$

to (5), we obtain

$$
\begin{gathered}
\mathcal{F}=-\lim _{K, L \rightarrow \infty} \lim _{u \rightarrow 0} \frac{\partial}{\partial u} \Xi_{K}^{(u)}, \\
\Xi_{K}^{(u)}=\frac{1}{K} \ln \mathrm{E}\left\{[p(\overrightarrow{\boldsymbol{y}} \mid \mathcal{S}, \mathcal{H})]^{u} \mid \mathcal{H}\right\} .
\end{gathered}
$$

We assume that the limit with respect to $K, L$ and the operation with respect to $u$ are interchangeable. Then, (7) becomes

$$
\mathcal{F}=-\lim _{u \rightarrow 0} \frac{\partial}{\partial u} \lim _{K, L \rightarrow \infty} \Xi_{K}^{(u)} .
$$

Further, we assume that the result for positive integers $u$ is valid for real number $u$ in the calculation of (9).

We define $N$-dimensional vectors $\boldsymbol{v}^{\alpha}$ as

$$
\boldsymbol{v}^{\alpha}=\frac{1}{\sqrt{\beta}} \sum_{k=1}^{K} s^{k} \boldsymbol{H}^{k} \boldsymbol{x}^{k, \alpha}, \quad \alpha=0, \ldots, u,
$$

where $\boldsymbol{x}^{k, 0}$ and $\boldsymbol{x}^{k, \alpha}=\left(x_{1}^{k, \alpha}, \ldots, x_{M_{k}}^{k, \alpha}\right)^{T}$ are the original data symbol vector and the replicated data symbol vector for the $k$ th user, respectively. $\left\{\boldsymbol{x}^{k, \alpha}, \alpha=0, \ldots, u\right\}$ are i.i.d. random variables following $p\left(\boldsymbol{x}^{k}\right)$ and $\left\{s^{k} ; k=1, \ldots, K\right\}$ are i.i.d. random variables following $p\left(s_{l}^{k}\right)$. Then, the expectation of $[p(\overrightarrow{\boldsymbol{y}} \mid \mathcal{S}, \mathcal{H})]^{u}$ with respect to $\overrightarrow{\boldsymbol{y}}$ and $\mathcal{S}$ is given by

$$
\begin{gathered}
\mathrm{E}\left\{[p(\overrightarrow{\boldsymbol{y}} \mid \mathcal{S}, \mathcal{H})]^{u} \mid \mathcal{H}\right\}=\mathrm{E}\left\{\exp \left[L G_{K}^{(u)}(\overrightarrow{\mathcal{X}}, \mathcal{H})\right] \mid \mathcal{H}\right\}, \\
G_{K}^{(u)}(\overrightarrow{\mathcal{X}}, \mathcal{H})=\ln \mathrm{E}_{\boldsymbol{S}}\left[\int \prod_{\alpha=0}^{u} \mathrm{e}^{-\frac{1}{\sigma^{2}}\left\|\boldsymbol{y}-\sqrt{\beta} \boldsymbol{v}^{\alpha}\right\|^{2}} \boldsymbol{d y}\right] \\
-N(u+1) \ln \left(\pi \sigma^{2}\right),
\end{gathered}
$$

where $\overrightarrow{\mathcal{X}}$ is defined as $\left\{\boldsymbol{x}^{k, \alpha} ; k=0, \ldots, K, \alpha=0, \ldots, u\right\}$ and $\boldsymbol{S}$ repserents $\left\{s^{k} ; k=1, \ldots, K\right\}$. When $K$ and $L$ are sufficiently large with their ratio fixed, due to the central limit theorem, $\boldsymbol{v}=\left(\boldsymbol{v}^{0^{T}}, \ldots, \boldsymbol{v}^{u T}\right)^{T}$ conditioned on $\overrightarrow{\mathcal{X}}$ and $\mathcal{H}$ follows approximately the zero-mean circularly symmetric complex Gaussian distribution with the covariance matrix

$$
\mathcal{Q}=\frac{1}{K} \sum_{k=1}^{K} \boldsymbol{w}^{k} \boldsymbol{w}^{k^{*}}
$$

where $\boldsymbol{w}^{k}$ is defined as $\left[\left(\boldsymbol{H}^{k} \boldsymbol{x}^{k, 0}\right)^{T}, \ldots,\left(\boldsymbol{H}^{k} \boldsymbol{x}^{k, u}\right)^{T}\right]^{T}$. Then, we can evaluate (11) and (12) as

$$
\Xi_{K}^{(u)}=\frac{1}{K} \ln \mathrm{E}\left\{\mathrm{e}^{K \beta^{-1} G^{(u)}(\mathcal{Q})}\right\}+\mathcal{O}\left(K^{-1}\right),
$$

$G^{(u)}(\mathcal{Q})=-\ln \operatorname{det}(\boldsymbol{I}+\boldsymbol{\Sigma} \mathcal{Q})-N u \ln \left(\pi \sigma^{2}\right)-N \ln (1+u)$,

where $\boldsymbol{\Sigma}$ is defined as

$$
\boldsymbol{\Sigma}=\frac{\beta}{\sigma^{2}(1+u)}\left[\begin{array}{cc}
u & -\boldsymbol{e}_{u}^{T} \\
-\boldsymbol{e}_{u} & (1+u) \boldsymbol{I}_{u}-\boldsymbol{e}_{u} \boldsymbol{e}_{u}^{T}
\end{array}\right] \otimes \boldsymbol{I}_{N},
$$

where $\boldsymbol{e}_{u}$ is the $u$-dimensional vector whose elements are all one and $\otimes$ represents the Kronecker product.

With a Hermitian matrix $\tilde{\mathcal{Q}}$ we define the moment generating function of the data symbols of the $k$ th user as

$$
\mathcal{M}_{k}^{(u)}(\tilde{\mathcal{Q}})=\mathrm{E}_{\mathcal{X}^{k}}\left\{\exp \left[\operatorname{tr}\left(\tilde{\mathcal{Q}} \boldsymbol{w}^{k} \boldsymbol{w}^{k^{*}}\right)\right]\right\},
$$

where $\mathcal{X}^{k}$ represents $\left\{x_{m_{k}}^{k, \alpha} ; m_{k}=1, \ldots, M_{k}, \alpha=0, \ldots, u\right\}$. Since $\mathcal{Q}$ satisfies the large deviation principle, with the saddle point method, (14) is evaluated as

$$
\lim _{K, L \rightarrow \infty} \Xi_{K}^{(u)}=\sup _{\mathcal{Q}}\left[\beta^{-1} G^{(u)}(\mathcal{Q})-I^{(u)}(\mathcal{Q})\right],
$$

where the rate function $I^{(u)}(\mathcal{Q})$ is given by

$$
I^{(u)}(\mathcal{Q})=\sup _{\tilde{\mathcal{Q}}}\left[\operatorname{tr}(\tilde{\mathcal{Q}} \mathcal{Q})-\lim _{K \rightarrow \infty} \frac{1}{K} \sum_{k=1}^{K} \ln \mathcal{M}_{k}^{(u)}(\tilde{\mathcal{Q}})\right] .
$$

Differentiating (18) and (19) with respect to $\mathcal{Q}$ and $\tilde{\mathcal{Q}}$, respectively, we obtain the following equations giving extrema of (18) and (19):

$$
\begin{gathered}
\tilde{\mathcal{Q}}^{\mathrm{s}}=-\beta^{-1}\left(\boldsymbol{I}+\boldsymbol{\Sigma} \mathcal{Q}^{\mathrm{s}}\right)^{-1} \boldsymbol{\Sigma} \\
\mathcal{Q}^{\mathrm{s}}=\lim _{K \rightarrow \infty} \frac{1}{K} \sum_{k=1}^{K} \frac{1}{\mathcal{M}_{k}^{(u)}\left(\tilde{\mathcal{Q}}^{\mathrm{s}}\right)} \mathrm{E}_{\mathcal{X}^{k}}\left[\boldsymbol{w}^{k} \boldsymbol{w}^{k^{*}} \mathrm{e}^{\left.\operatorname{tr}\left(\tilde{\mathcal{Q}}^{\mathrm{s}} \boldsymbol{w}^{k} \boldsymbol{w}^{k^{*}}\right)\right]}\right.
\end{gathered}
$$

Differentiating (18) with respect to $u$ and substituting (20) to it, we can evaluate (9) as

$$
\mathcal{F}=-\lim _{u \rightarrow 0}\left[\beta^{-1} \frac{\partial G^{(u)}}{\partial u}\left(\mathcal{Q}^{\mathrm{s}}\right)-\frac{\partial I^{(u)}}{\partial u}\left(\mathcal{Q}^{\mathrm{s}}\right)\right] .
$$

To evaluate the solution of (20) and (21) in the $u \rightarrow 0$ limit analytically we assume that the replica symmetry holds, i.e., 
$\mathcal{Q}^{\mathrm{s}}$ and $\tilde{\mathcal{Q}}^{\mathrm{s}}$ are invariant under exchange of non-zero replica indexes. Then, $\mathcal{Q}^{\mathrm{s}}$ and $\tilde{\mathcal{Q}}^{\mathrm{s}}$ can be written as

$$
\begin{aligned}
& \mathcal{Q}^{\mathrm{s}}=\left(\begin{array}{cc}
\boldsymbol{Q}^{0} & \boldsymbol{e}_{u}^{T} \otimes \boldsymbol{M} \\
\boldsymbol{e}_{u} \otimes \boldsymbol{M}^{*} & \boldsymbol{I}_{u} \otimes\left(\boldsymbol{Q}^{1}-\boldsymbol{Q}\right)+\boldsymbol{e}_{u} \boldsymbol{e}_{u}^{T} \otimes \boldsymbol{Q}
\end{array}\right), \\
& \tilde{\mathcal{Q}}^{\mathrm{s}}=\left(\begin{array}{cc}
\tilde{\boldsymbol{Q}}^{0} & \boldsymbol{e}_{u}^{T} \otimes \tilde{\boldsymbol{M}} \\
\boldsymbol{e}_{u} \otimes \tilde{\boldsymbol{M}}^{*} & \boldsymbol{I}_{u} \otimes\left(\tilde{\boldsymbol{Q}}^{1}-\tilde{\boldsymbol{Q}}\right)+\boldsymbol{e}_{u} \boldsymbol{e}_{u}^{T} \otimes \tilde{\boldsymbol{Q}}
\end{array}\right),
\end{aligned}
$$

where $\boldsymbol{M}, \tilde{\boldsymbol{M}}$ are $N$-by- $N$ matrices and $\boldsymbol{Q}^{0}, \tilde{\boldsymbol{Q}}^{0}, \boldsymbol{Q}^{1}, \tilde{\boldsymbol{Q}}^{1}$, $\boldsymbol{Q}$, and $\tilde{\boldsymbol{Q}}$ are $N$-by- $N$ Hermitian matrices. By solving (20) we have in the $u \rightarrow 0$ limit

$$
\tilde{\boldsymbol{Q}}^{0}=\mathbf{0}, \tilde{\boldsymbol{M}}=\boldsymbol{R}^{-1}, \tilde{\boldsymbol{Q}}^{1}=\tilde{\boldsymbol{Q}}-\tilde{\boldsymbol{M}}, \tilde{\boldsymbol{Q}}=\boldsymbol{R}^{-1} \boldsymbol{R}_{0} \boldsymbol{R}^{-1}
$$

where $\boldsymbol{R}_{0}, \boldsymbol{R}$ are defined as

$$
\begin{aligned}
\boldsymbol{R}_{0} & =\sigma^{2} \boldsymbol{I}_{N}+\beta\left(\boldsymbol{Q}^{0}-\boldsymbol{M}-\boldsymbol{M}^{*}+\boldsymbol{Q}\right), \\
\boldsymbol{R} & =\sigma^{2} \boldsymbol{I}_{N}+\beta\left(\boldsymbol{Q}^{1}-\boldsymbol{Q}\right) .
\end{aligned}
$$

It is straightforward to confirm that $\boldsymbol{R}_{0}, \boldsymbol{R}$ are positive definite.

We move on to calculating the moment generating function (17). $\boldsymbol{R}_{0}$ can be decomposed into the product of two nonsingular matrices, i.e., $\boldsymbol{R}_{0}=\sqrt{\boldsymbol{R}_{0}}{\sqrt{\boldsymbol{R}_{0}}}^{*}$. From (25) the moment generating function (17) is evaluated as

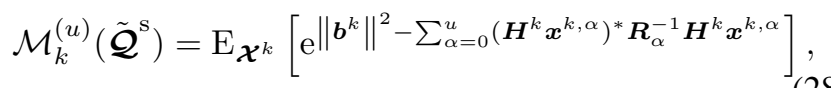

where $\boldsymbol{R}_{\alpha}=\boldsymbol{R}$ for $\alpha=1, \ldots, u$, and where $\boldsymbol{b}^{k}$ is defined as

$$
\boldsymbol{b}^{k}=\left(\sqrt{\boldsymbol{R}_{0}}\right)^{-1} \boldsymbol{H}^{k} \boldsymbol{x}^{k, 0}+{\sqrt{\boldsymbol{R}_{0}}}^{*} \boldsymbol{R}^{-1} \sum_{\alpha=1}^{u} \boldsymbol{H}^{k} \boldsymbol{x}^{k, \alpha} .
$$

By applying the transform:

$$
\mathrm{e}^{\left\|\boldsymbol{b}^{k}\right\|^{2}}=\int \frac{1}{\pi^{N} \operatorname{det} \boldsymbol{R}_{0}} \mathrm{e}^{-\boldsymbol{y}^{k *} \boldsymbol{R}_{0}^{-1} \boldsymbol{y}^{k}+2 \Re\left(\boldsymbol{b}^{k *}\left(\sqrt{\boldsymbol{R}_{0}}\right)^{-1} \boldsymbol{y}^{k}\right)} \boldsymbol{d} \boldsymbol{y}^{k},
$$

we obtain

$$
\begin{gathered}
\mathcal{M}_{k}^{(u)}\left(\tilde{\mathcal{Q}}^{\mathrm{s}}\right)=\int \mathrm{E}_{\boldsymbol{x}^{k}}\left[p\left(\boldsymbol{y}^{k} \mid \boldsymbol{x}^{k}, \boldsymbol{H}^{k} ; \boldsymbol{R}_{0}\right)\right] \\
\left\{\frac{\mathrm{E}_{\boldsymbol{x}^{k}}\left[p\left(\boldsymbol{y}^{k} \mid \boldsymbol{x}^{k}, \boldsymbol{H}^{k} ; \boldsymbol{R}\right)\right]}{p\left(\boldsymbol{y}^{k} \mid \mathbf{0}, \boldsymbol{H}^{k} ; \boldsymbol{R}\right)}\right\}^{u} \boldsymbol{d} \boldsymbol{y}^{k},
\end{gathered}
$$

where $p\left(\boldsymbol{y}^{k} \mid \boldsymbol{x}^{k}, \boldsymbol{H}^{k} ; \boldsymbol{R}\right)$ is defined as

$$
p\left(\boldsymbol{y}^{k} \mid \boldsymbol{x}^{k}, \boldsymbol{H}^{k} ; \boldsymbol{R}\right)=\frac{\mathrm{e}^{-\left(\boldsymbol{y}^{k}-\boldsymbol{H}^{k} \boldsymbol{x}^{k}\right)^{*} \boldsymbol{R}^{-1}\left(\boldsymbol{y}^{k}-\boldsymbol{H}^{k} \boldsymbol{x}^{k}\right)}}{\pi^{N} \operatorname{det} \boldsymbol{R}} .
$$

Assuming that $\boldsymbol{R}_{0}$ is equal to $\boldsymbol{R}$, from (21) we obtain

$$
\begin{gathered}
\boldsymbol{Q}^{0}-\boldsymbol{M}-\boldsymbol{M}^{*}+\boldsymbol{Q}=\boldsymbol{Q}^{1}-\boldsymbol{Q}=\lim _{K \rightarrow \infty} \frac{1}{K} \sum_{k=1}^{K} \mathcal{E}^{k}, \\
\mathcal{E}^{k}=\mathrm{E}\left[\boldsymbol{H}^{k}\left(\boldsymbol{x}^{k}-\left\langle\boldsymbol{x}^{k}\right\rangle_{\mathrm{MIMO}_{1}}\right)\left(\boldsymbol{x}^{k}-\left\langle\boldsymbol{x}^{k}\right\rangle_{\mathrm{MIMO}_{1}}\right)^{*} \boldsymbol{H}^{k^{*}}\right],
\end{gathered}
$$

where $\langle\cdot\rangle_{\mathrm{MIMO}_{1}}$ is defined as

$$
\left\langle\boldsymbol{x}^{k}\right\rangle_{\mathrm{MIMO}_{1}}=\frac{\mathrm{E}_{\boldsymbol{x}^{k}}\left[\boldsymbol{x}^{k} p\left(\boldsymbol{y}^{k} \mid \boldsymbol{x}^{k}, \boldsymbol{H}^{k} ; \boldsymbol{R}\right)\right]}{\mathrm{E}_{\boldsymbol{x}^{k}}\left[p\left(\boldsymbol{y}^{k} \mid \boldsymbol{x}^{k}, \boldsymbol{H}^{k} ; \boldsymbol{R}\right)\right]} .
$$

Note that $\mathcal{E}^{k}$ is averaged with respect to $\boldsymbol{H}^{k}$ due to the law of large numbers. Substituting (33) to (26) and (27), we obtain the fixed-point equation

$$
\boldsymbol{R}=\sigma^{2} \boldsymbol{I}_{N}+\beta \lim _{K \rightarrow \infty} \frac{1}{K} \sum_{k=1}^{K} \mathcal{E}^{k},
$$

which is the extension of the Tse-Hanly equation [9] to the case of the MIMO-CDMA channel.

Calculating $G^{(u)}\left(\mathcal{Q}^{\mathrm{s}}\right), I^{(u)}\left(\mathcal{Q}^{\mathrm{s}}\right)$ and differentiating them with respect to $u$, we can evaluate $\mathcal{F}$ as

$$
\begin{aligned}
\frac{\beta}{\ln 2} \mathcal{F}=\lim _{K \rightarrow \infty} & \frac{1}{K} \sum_{k=1}^{K} \beta M_{k} \mathcal{C}_{\mathrm{MIMO}_{1}}^{k}+N \log \left(\pi \sigma^{2} \mathrm{e}\right) \\
+ & \operatorname{KL}\left(\mathcal{C N}\left(\mathbf{0}, \sigma^{2} \boldsymbol{I}_{N}\right) \| \mathcal{C N}(\mathbf{0}, \boldsymbol{R})\right),
\end{aligned}
$$

where $\operatorname{KL}(\cdot \| \cdot)$ represents the Kullback-Leibler divergence, and where $\mathcal{C}_{\mathrm{MIMO}_{1}}^{k}$ is defined as

$$
\mathcal{C}_{\mathrm{MIMO}_{1}}^{k}=\frac{1}{M_{k}} \mathrm{E}\left\{\log \frac{p\left(\boldsymbol{y}^{k} \mid \boldsymbol{x}^{k}, \boldsymbol{H}^{k} ; \boldsymbol{R}\right)}{\mathrm{E}_{\boldsymbol{x}^{k}}\left[p\left(\boldsymbol{y}^{k} \mid \boldsymbol{x}^{k}, \boldsymbol{H}^{k} ; \boldsymbol{R}\right)\right]} \mid \boldsymbol{H}^{k}\right\} .
$$

Note that (37) depends only on $\boldsymbol{R}$. In the case that there exist multiple solutions of (36), one should choose the solution achieving the supremum of (18) in a neighborhood of $u=0$, i.e., the solution minimizing (37).

From the above mentioned analysis we claim that the spectral efficiency of the MIMO-CDMA channel is evaluated as

$$
\begin{array}{r}
\mathcal{C}_{\mathrm{MIMO}-\mathrm{CDMA}}=\lim _{K \rightarrow \infty} \frac{1}{K} \sum_{k=1}^{K} \frac{\beta M_{k}}{\bar{M}} \mathrm{E}_{\boldsymbol{H}^{k}}\left[\mathcal{C}_{\mathrm{MIMO}_{1}}^{k}\right] \\
+\frac{1}{\bar{\mu} N} \mathrm{KL}\left(\mathcal{C N}\left(\mathbf{0}, \sigma^{2} \boldsymbol{I}_{N}\right) \| \mathcal{C N}(\mathbf{0}, \boldsymbol{R})\right) .
\end{array}
$$

$\mathrm{E}_{\boldsymbol{H}^{k}}\left[\mathcal{C}_{\mathrm{MIMO}_{1}}^{k}\right]$ can be interpreted as the spectral efficiency of the following single-user MIMO Gaussian channel for the $k$ th user:

$$
\boldsymbol{y}^{k}=\boldsymbol{H}^{k} \boldsymbol{x}^{k}+\boldsymbol{n}^{k}, \quad \boldsymbol{n}^{k} \sim \mathcal{C N}(\mathbf{0}, \boldsymbol{R}) .
$$

From the replica analysis for the moment sequence of the posterior mean estimator $\left\langle x_{m_{k}}^{k}\right\rangle_{\text {MIMO-CDMA }}=\mathrm{E}\left[x_{m_{k}}^{k} \mid \overrightarrow{\boldsymbol{y}}, \mathcal{S}, \mathcal{H}\right]$, (39) indicate that the MIMO-CDMA channel with the MMSE detector front end is decoupled into a bank of single-user MIMO channels with the MMSE detector front ends in the many-user limit. It is easy to extend this decoupling result to the cases of the linear MMSE detector or the matched filter.

\section{RePliCa ANALysis of MIMO}

So far we have not specified statistics of the elements of $\boldsymbol{H}^{k}$. In order to obtain a more concrete expression of (39), we consider a fading model with a limited number of scatterers [6]

$$
\boldsymbol{H}^{k}=\boldsymbol{\Phi}^{k^{*}} \boldsymbol{A}^{k} \boldsymbol{\Theta}^{k},
$$

where $\Theta^{k}$ is a $S_{k}$-by- $M_{k}$ steering matrix which describes the propagation from the transmit antennas of the $k$ th user 
to $S_{k}$ scattering objects between the $k$ th user and the receiver, where $\boldsymbol{A}^{k}=\operatorname{diag}\left(A_{1}^{k}, \ldots, A_{S_{k}}^{k}\right)$ is a $S_{k}$-by- $S_{k}$ diagonal matrix which accounts for attenuation at the scattering objects between the $k$ th user and the receiver, and where $\boldsymbol{\Phi}^{k}$ is a $S_{k^{-}}$ by- $N$ steering matrix which describes the propagation from the scattering objects between the $k$ th user and the receiver to the receive antennas of the receiver. We assume that the elements of $\boldsymbol{\Phi}^{k}$ and $\boldsymbol{\Theta}^{k}$ are i.i.d. random variables with the variances $1 / N$ and $1 / S_{k}$, respectively, and that the elements of $\boldsymbol{A}^{k}$ are independent random variables subject to the normalization $\mathrm{E}\left[\operatorname{tr}\left(\boldsymbol{A}^{k} \boldsymbol{A}^{k^{*}}\right)\right]=S_{k}$.

It is difficult to evaluate (34) and (38) analytically except in special cases, e.g., the data symbol vector of the $k$ th user $\boldsymbol{x}^{k}$ follows a circularly symmetric complex Gaussian distribution or the users and the receiver have a few numbers of the antennas. Thus, we evaluate (34) and (38) using the replica method in the many-antenna limit where $M_{k}, S_{k}$, and $N$ tend to infinity with their ratios $\rho_{k}=S_{k} / N, \gamma_{k}=M_{k} / S_{k}$ fixed. One might think that the assumption of the many-antenna limit is impractical, but it can be a good approximate approach to systems with a few antennas if the elements of $\boldsymbol{x}^{k}$ and $\boldsymbol{H}^{k}$ follow circularly symmetric complex Gaussian distributions [1]. On the other hand, the spectral efficiency is not invariant under exchange of the order of the many-user limit and the many-antenna limit. In the MIMO-CDMA literature, however, it may be reasonable to take the many-user limit first.

By regarding $\boldsymbol{\Phi}^{k^{*}}$ and $\boldsymbol{A}^{k} \boldsymbol{\Theta}^{k} \boldsymbol{x}^{k}$ as the channel matrix and the data symbol vector, respectively, we can evaluate the expectation of (38) as

$$
\begin{array}{r}
\lim \mathrm{E}_{\boldsymbol{H}^{k}}\left[\mathcal{C}_{\mathrm{MIMO}_{1}}^{k}\right]=\lim _{S_{k}, M_{k} \rightarrow \infty} \mathrm{E}_{\boldsymbol{A}^{k} \boldsymbol{\Theta}^{k}}\left[\mathcal{C}_{\mathrm{MIMO}_{2}}^{k}\right] \\
+\lim _{N \rightarrow \infty} \frac{1}{\mu_{k} N} \mathrm{KL}\left(\mathcal{C N}(\mathbf{0}, \boldsymbol{R}) \| \mathcal{C N}\left(\mathbf{0}, \boldsymbol{W}^{k}\right)\right),
\end{array}
$$

where $\mu_{k}=M_{k} / N$, where lim represents the many-antenna limit, and where $\mathcal{C}_{\mathrm{MIMO}_{2}}^{k}$ is the spectral efficiency of the following MIMO channel for the $k$ th user:

$$
\tilde{\boldsymbol{y}}^{k}=\boldsymbol{A}^{k} \boldsymbol{\Theta}^{k} \boldsymbol{x}^{k}+\tilde{\boldsymbol{n}}^{k}, \quad \tilde{\boldsymbol{n}}^{k} \sim \mathcal{C N}\left(\mathbf{0}, \zeta^{k^{2}} \boldsymbol{I}_{S_{k}}\right) .
$$

$\zeta^{k^{2}}$ and $\boldsymbol{W}^{k}$ satisfy the following fixed-point equations:

$$
\zeta^{k^{-2}}=\lim _{N \rightarrow \infty} \frac{1}{N} \operatorname{tr}\left[\boldsymbol{W}^{k^{-1}}\right], \boldsymbol{W}^{k}=\boldsymbol{R}+\rho_{k} \mathcal{E}_{\mathrm{MIMO}_{2}}^{k} \boldsymbol{I}_{N},
$$

$\mathcal{E}_{\mathrm{MIMO}_{2}}^{k}=\lim _{M_{k}, S_{k} \rightarrow \infty} \frac{1}{S_{k}} \mathrm{E}\left[\left\|\boldsymbol{A}^{k} \boldsymbol{\Theta}^{k}\left(\boldsymbol{x}^{k}-\left\langle\boldsymbol{x}^{k}\right\rangle_{\mathrm{MIMO}_{2}}\right)\right\|^{2}\right]$,

where $\left\langle\boldsymbol{x}^{k}\right\rangle_{\mathrm{MIMO}_{2}}=\mathrm{E}\left[\boldsymbol{x}^{k} \mid \tilde{\boldsymbol{y}}^{k}, \boldsymbol{A}^{k} \boldsymbol{\Theta}^{k}\right]$ and where the operator $\mathrm{E}$ in (45) represents the expectation with respect to $p\left(\tilde{\boldsymbol{y}}^{k}, \boldsymbol{x}^{k}, \boldsymbol{A}^{k} \boldsymbol{\Theta}^{k}\right)$. In the case that there exist multiple solutions of (44) and (45), one should choose the solution minimizing the spectral efficiency (42).

To evaluate (34) we define a quantity $\tilde{\mathcal{F}}_{\mathrm{MIMO}_{1}}^{k}$ as

$$
\tilde{\mathcal{F}}_{\mathrm{MIMO}_{1}}^{k}=\lim \frac{1}{S_{k}} \ln \mathrm{E}\left[\tilde{Z}_{\mathrm{MIMO}_{1}}^{(u)}\left(\boldsymbol{y}^{k}, \boldsymbol{H}^{k} ; \boldsymbol{\Omega}\right)\right],
$$

$$
\tilde{Z}_{\mathrm{MIMO}_{1}}^{(u)}=\mathrm{E}_{\boldsymbol{\mathcal { X }}^{k}}\left\{\mathrm{e}^{\operatorname{tr}\left[\boldsymbol{\Omega}^{T} \boldsymbol{F}\right]} \prod_{\alpha=0}^{u} p\left(\boldsymbol{y}^{k} \mid \boldsymbol{x}^{k, \alpha}, \boldsymbol{H}^{k} ; \boldsymbol{R}\right)\right\},
$$

where $\boldsymbol{F}$ is defined as

$$
\boldsymbol{F}\left(\boldsymbol{\mathcal { X }}^{k}, \boldsymbol{H}^{k}\right)=S_{k} \boldsymbol{H}^{k}\left(\boldsymbol{x}^{k}-\boldsymbol{x}^{k, 1}\right)\left(\boldsymbol{x}^{k}-\boldsymbol{x}^{k, 2}\right)^{*} \boldsymbol{H}^{k^{*}} .
$$

Then, we obtain in the many-antenna limit [4]

$$
\lim \mathcal{E}^{k}=\left.\lim _{u \rightarrow 0} \frac{\partial}{\partial \Omega} \tilde{\mathcal{F}}_{\mathrm{MIMO}_{1}}\right|_{\boldsymbol{\Omega}=\mathbf{0}} .
$$

Calculating the right-hand side of (49), we can evaluate 34 as

$$
\lim \mathcal{E}^{k}=\boldsymbol{R}-\boldsymbol{R} \tilde{\boldsymbol{W}}^{k^{-1}} \boldsymbol{R},
$$

where $\tilde{\boldsymbol{W}}^{k}$ is a solution of (44) and (45). We have added a tilde to $\boldsymbol{W}^{k}$ in order to make clear that $\tilde{\boldsymbol{W}}^{k}$ need not be equal to $\boldsymbol{W}^{k}$ in (42). In the case that there exist multiple $\tilde{\boldsymbol{W}}^{k}$ one should choose the solution minimizing (42).

Furthermore, we can evaluate $\mathrm{E}\left[\mathcal{C}_{\mathrm{MIMO}_{2}}^{k}\right]$ and $\mathcal{E}_{\mathrm{MIMO}_{2}}^{k}$ by applying the above mentioned method again. On the assumption that $\left\{x_{m_{k}}^{k} ; m_{k}=1, \ldots, M_{k}\right\}$ are mutually independent, $\mathrm{E}\left[\mathrm{C}_{\mathrm{MIMO}_{2}}^{k}\right]$ in the many-antenna limit is given by [6], [7]

$$
\begin{array}{r}
\lim _{S_{k}, M_{k} \rightarrow \infty} \mathrm{E}\left[\mathcal{C}_{\mathrm{MIMO}_{2}}^{k}\right]=\lim _{M_{k} \rightarrow \infty} \frac{1}{M_{k}} \sum_{m_{k}=1}^{M_{k}} \mathcal{C}_{\mathrm{AWGN}}^{k, m_{k}} \\
+\lim _{S_{k} \rightarrow \infty} \frac{\gamma_{k}^{-1}}{S_{k}} \mathrm{E}_{\boldsymbol{A}^{k}}\left[\mathrm{KL}\left(\mathcal{C N}\left(\mathbf{0}, \zeta^{k^{2}} \boldsymbol{I}_{S_{k}}\right) \| \mathcal{C N}\left(\mathbf{0}, \boldsymbol{\Xi}^{k}\right)\right)\right],
\end{array}
$$

where $\mathcal{C}_{\mathrm{AWGN}}^{k, m_{k}}$ is the spectral efficiency of the scalar Gaussian channel

$$
y_{m_{k}}^{k}=x_{m_{k}}^{k}+n_{m_{k}}^{k}, \quad n_{m_{k}}^{k} \sim \mathcal{C N}\left(0, \xi^{k^{2}}\right) .
$$

$\xi^{k^{2}}$ and $\boldsymbol{\Xi}^{k}$ satisfy the following fixed-point equations:

$$
\begin{gathered}
\xi^{k^{-2}}=\lim _{S_{k} \rightarrow \infty} \frac{1}{S_{k}} \sum_{s_{k}=1}^{S_{k}} \mathrm{E}\left[\frac{\left|A_{s_{k}}^{k}\right|^{2}}{\zeta^{k^{2}}+\gamma_{k} \mathcal{E}_{\mathrm{AWGN}}^{k}\left|A_{s_{k}}^{k}\right|^{2}}\right] \\
\boldsymbol{\Xi}^{k}=\zeta^{k^{2}} \boldsymbol{I}_{S_{k}}+\gamma_{k} \mathcal{E}_{\mathrm{AWGN}}^{k} \boldsymbol{A}^{k} \boldsymbol{A}^{k^{*}} \\
\mathcal{E}_{\mathrm{AWGN}}^{k}=\lim _{M_{k} \rightarrow \infty} \frac{1}{M_{k}} \sum_{m_{k}=1}^{M_{k}} \mathrm{E}\left[\mid\left(x_{m_{k}}^{k}-\left\langle x_{m_{k}}^{k}\right\rangle_{\mathrm{AWGN}}\right)^{2}\right],
\end{gathered}
$$

where $\left\langle x_{m_{k}}^{k}\right\rangle_{\mathrm{AWGN}}=\mathrm{E}\left[x_{m_{k}}^{k} \mid y_{m_{k}}^{k}\right]$. In the case that there exist multiple solutions of (53), (54), and (55), one should choose the solution minimizing the spectral efficiency (51).

The result of a replica analysis claims that the moment sequence of $\left\langle x_{m_{k}}^{k}\right\rangle_{\text {MIMO-CDMA }}$ converges to the moment sequence of $\left\langle x_{m_{k}}^{k}\right\rangle_{\text {AWGN }}$ in the many-user and many-antenna limits. Since the MMSE detector is information lossless in the scalar Gaussian channel, $\mathcal{C}_{\mathrm{AWGN}}^{k, m_{k}}$ can be interpreted as the spectral efficiency of the MMSE detector in the MIMOCDMA channel.

On the other hand, (45) is evaluated as

$$
\mathcal{E}_{\mathrm{MIMO}_{2}}^{k}=\lim _{S_{k} \rightarrow \infty} \frac{1}{S_{k}} \sum_{s_{k}=1}^{S_{k}} \mathrm{E}\left[\zeta^{k^{2}}-\frac{\zeta^{k^{4}}}{\zeta^{k^{2}}+\gamma_{k} \tilde{\mathcal{E}}_{\mathrm{AWGN}}^{k}\left|A_{s_{k}}^{k}\right|^{2}}\right],
$$


where $\tilde{\mathcal{E}}_{\mathrm{AWGN}}^{k}$ is a solution of (53), (54), and (55). In the case that there exist multiple $\tilde{\mathcal{E}}_{\mathrm{AWGN}}^{k}$ one should choose the solution minimizing (51). We can obtain the same fixed-point equations for $\zeta^{k^{2}}$ and $\xi^{k^{2}}$ by means of calculating the expectation of $\mathcal{C}_{\mathrm{MIMO}_{1}}^{k}$ with respect to $\Theta^{k}$ and then evaluating the asymptotic distribution of singular values of $\boldsymbol{\Phi}^{k}$.

We can easily confirm that the spectral efficiency (39) coincides with the spectral efficiency of the i.i.d. Rayleigh fading MIMO-CDMA channel in the case that scattering is very rich. In the $\gamma_{k} \rightarrow 0$ limit with $\mu_{k}=\rho_{k} \gamma_{k}$ fixed, (53) reduces to $\xi^{k^{2}}=\zeta^{k^{2}}$. Expanding (56) with respect to $\gamma_{k}$ and substituting it to (44), we obtain

$\zeta^{k^{-2}}=\lim _{N \rightarrow \infty} \frac{1}{N} \operatorname{tr}\left[\boldsymbol{W}^{k^{-1}}\right], \boldsymbol{W}^{k}=\boldsymbol{R}+\mu_{k} \mathcal{E}_{\mathrm{AWGN}}\left(\zeta^{k^{2}}\right) \boldsymbol{I}_{N}$

(57) coincides with the fixed-point equation for $\zeta^{k^{2}}$ and $\boldsymbol{W}^{k}$ in the case of the i.i.d. Rayleigh fading. Hence, (39) converges to the spectral efficiency of the i.i.d. Rayleigh fading MIMOCDMA channel in the $\gamma_{k} \rightarrow 0$ limit.

The above mentioned results indicate that the MIMO channel (40) with the MMSE detector front end is decoupled into the bank of scalar Gaussian channels with the MMSE detector front ends in the many-antenna limit even if the number of the scatterers is limited. It is easy to extend this decoupling result to the cases of the linear MMSE detector or the matched filter.

\section{Numerical EVALUATION}

To evaluate the spectral efficiency numerically we consider the case that $\rho=\rho_{k}, \gamma=\gamma_{k},\left\{x_{m_{k}}^{k}\right\}$ are i.i.d. random variables with the variance $P=\mathrm{E}\left[\left|x_{m_{k}}^{k}\right|^{2}\right]$, and $\left|A_{s_{k}}^{k}\right|^{2}=1$ with probability $1 . \mu=\rho \gamma$ represents the ratio of the number of transmit antennas to the number of receive antennas, i.e., $\mu=M / N$. We denote the received signal-to-noise ratio per transmit antenna by $\mathrm{SNR}=P / \sigma_{0}^{2}$. Figure 1] displays the spectral efficiency (39) versus SNR for QPSK modulation (4QAM). We find that the spectral efficiency of the MIMOCDMA channel with QPSK modulation is very close to the capacity but there exists a large gap between the spectral efficiency of the MMSE detector and the capacity in the highly loaded system. The degradation of the spectral efficiency due to the decrease of scatterers is shown in Fig. 2 In this case, an interesting observation is that the spectral efficiency does not degrade so much compared with the rich scattered environment $(\gamma \rightarrow 0)$ even when the number of scatterers is comparable with the number of the antennas.

\section{CONCLUSION}

We evaluated the spectral efficiency of the uplink of the MIMO-CDMA channel using the replica method. The main result is that the hierarchical decoupling principle holds in the MIMO-CDMA channel, i.e., the MIMO-CDMA channel is decoupled into the bank of single-user MIMO channels in the many-user limit. The resulting single-user MIMO channel is further decoupled into the bank of scalar Gaussian channels in the many-antenna limit for the fading model with a limited number of scatterers. We found numerically that the spectral efficiency of the MIMO-CDMA channel with QPSK modulation is very close to the capacity but there exists a large gap between the spectral efficiency of the MMSE detector and the capacity in a highly loaded system.

\section{ACKNOWLEDGMENT}

The authors are grateful for supports from Grant-in-Aid for Scientific Research on Priority Areas 18079010, MEXT, Japan.

\section{REFERENCES}

[1] D. Tse and P. Viswanath. Fundamentals of Wireless Communication Cambridge University Press, 2005.

[2] A. Mantravadi et al. IEEE J. Select. Areas Com., Vol. 21, p. 382, 2003.

[3] Z. Ni and D. Li. Wirel. Commun. Mob. Comput., Vol. 5, p. 35, 2005.

[4] T. Tanaka. IEEE Trans. I. T., Vol. 48, p. 2888, 2002.

[5] A. L. Moustakas et al. IEEE Trans. I. T., Vol. 49, p. 2545, 2003.

[6] R. R. Müller. IEEE Trans. Signal Processing, Vol. 51, p. 2821, 2003.

[7] C.-K. Wen et al. IEEE Trans. Commun., Vol. 54, p. 349, 2006.

[8] D. Guo and S. Verdú. IEEE Trans. I. T., Vol. 51, p. 1983, 2005.

[9] D. Tse and S. V. Hanly. IEEE Trans. I. T., Vol. 45, p. 641, 1999.

[10] S. Verdú and S. Shamai. IEEE Trans. I. T., Vol. 45, p. 622, 1999.

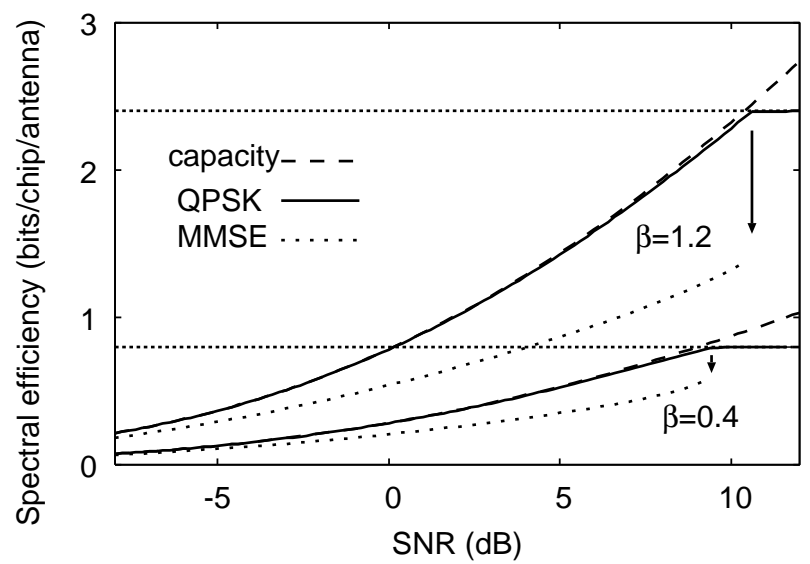

Fig. 1. Spectral efficiency of the MIMO-CDMA channel versus SNR. $\mu=1.0$ and $\gamma=1.0$.

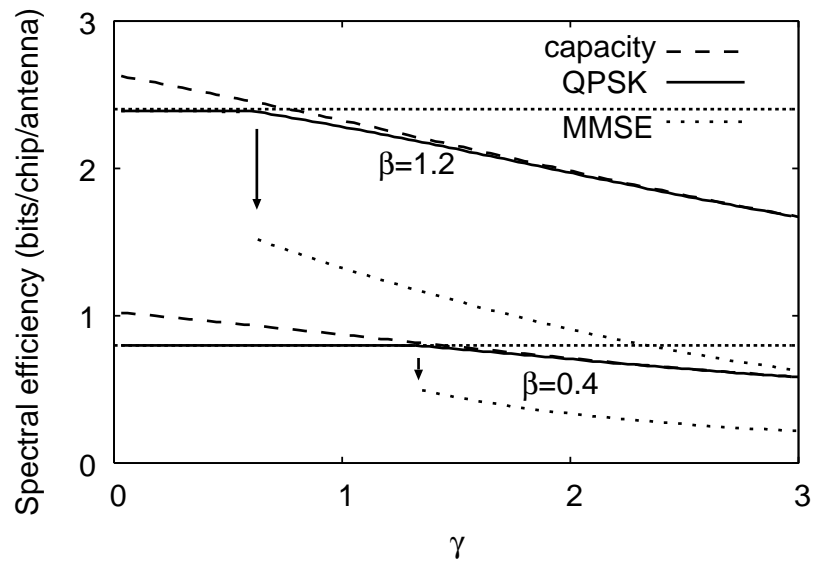

Fig. 2. Spectral efficiency of the MIMO-CDMA channel for QPSK modulation versus $\gamma \cdot \mu=1.0$ and SNR $=10 \mathrm{~dB}$. The spectral efficiency coincides with the spectral efficiency of the i.i.d. Rayleigh fading MIMOCDMA channel in the $\gamma \rightarrow 0$ limit. 